\title{
Structural insights into glutathione-mediated activation of the master regulator PrfA in Listeria monocytogenes
}

\section{Dear Editor}

Listeria monocytogenes is a Gram-positive and facultative intracellular bacterial pathogen with two distinct lifestyles: saprophytic in the soil and parasitic in host cells (Freitag et al., 2009). L. monocytogenescan cause a foodborne infection characterized by bacteremia, meningoencephalitis, abortion or neonatal sepsis and a high case-fatality rate (Freitag et al., 2009). In relation to pathogenesis, the expression of most virulence genes in L. monocytogenes is regulated by the master regulator PrfA, which is a member of the Crp/Fnr family of site-specific DNA-binding transcription regulators (Freitag et al., 2009). The absolute requirement of PrfA for pathogenesis was demonstrated utilizing $L$. monocytogenesstrains with deletions or loss-of-function mutations within the prfA gene (Chakraborty et al., 1992; Freitag et al., 1993; Leimeister-Wachter et al., 1990). PrfA activates transcription by binding to a palindromic promoter element termed the PrfA box (tTAACanntGTtAa). Very recently, glutathione $(\mathrm{GSH})$, either generated by bacteria or derived from host cells, was found to be the essential small molecule cofactor of PrfA through allosteric binding to the protein (Reniere et al., 2015). PrfA ${ }^{\mathrm{G} 145 \mathrm{~S}}$, the most well studied constitutively active mutant, was found to be able to completely bypass the requirement for glutathione during infection (Reniere et al., 2015).

Infection by $L$. monocytogenes can be detected by the cytosolic DNA sensing pathway of the host cell, thereby inducing the expression of type I interferons (IFNs) (Hansen et al., 2014). Although type I IFNs are well known for their ability to protect the host from viral infections and some bacterial infections, these pleiotropic cytokines are found to be able to exacerbate infections by $L$. monocytogenes (Rayamajhi et al., 2010). It was also found that $L$. monocytogenes is intrinsically resistant to broad spectrum cephalosporin antibiotics, which are commonly used in the treatment of bacterial infections (Krawczyk-Balska and Markiewicz, 2015). Thus a better understanding of PrfA regulation may give us an alternative strategy to control infection. Despite extensive genetic and biochemical research, the detailed molecular mechanism of PrfA activation and regulation is still unclear due to the lack of structures of PrfA bound to DNA and cofactor. In this study, we determined the crystal structures of PrfA-DNA binary and PrfA-DNA-GSH ternary complexes, thereby providing new insights into the mechanism of PrfA-mediated gene regulation.

We have co-crystallized PrfA bound to an intact 28-bp complementary dsDNA (plus 1-nt 5 ' overhang at either end) and solved the structure of the binary complex at $2.93 \AA$ resolution (X-ray statistics in Table S1). The complex contains one PrfA dimer bound to one dsDNA molecule and exhibits an intramolecular 2-fold pseudosymmetry (Fig. 1A). Each PrfA monomer consists of an $\mathrm{N}$-terminal domain (aa 1-108), a long a-helical linker (aa 109-1137), and a helix-loop-helix-containing C-terminal domain (aa 138-1237) (Fig. 1A). The overall DNA bend is $\sim 45^{\circ}$ in the PrfA-DNA complex (Fig. 1A), which is significantly different from the previously reported bend values of approx. $80^{\circ}$ to $90^{\circ}$ for Crp-DNA (Benoff et al., 2002; Schultz et al., 1991) and CprK-DNA (Levy et al., 2008) complexes (Fig. S1A). Given the high quality electron density map for the DNA in the complex, we could readily build each nucleotide into the map (Fig. S1B). The intermolecular contacts between PrfA dimer and DNA (summarized in Fig. 1B) contain both specific interactions with bases and nonspecific interactions with sugar-phosphate backbone. The second helix of the helix-loop-helix motif penetrates into the major groove of the DNA, with S184 and R188 forming direct hydrogen bonds with the bases of T20 and G18, respectively (Fig. 1C). The majority of the intermolecular contacts are between PrfA and the sugar-phosphate backbone of the DNA (Fig. 1D and 1E). The superimposed structures of PrfA in DNA-bound state with either PrfA or PrfA ${ }^{G 145 S}$ in free state (Eiting et al., 2005) are shown in Fig. S1C and S1D, respectively. As expected, PrfA in the DNA-bound state is highly similar to the constitutively active mutant $\operatorname{PrfA}^{\mathrm{G} 145 \mathrm{~S}}$ (Figs. S1D and $1 \mathrm{G}$ ), while comparison with wild type PrfA shows significant differences in the helix-loop-helix motif and the linker region (Figs. S1C and $1 \mathrm{~F}$ ).

We have solved the structure of the PrfA-DNA-GSH ternary complex at $2.99 \AA$ (X-ray statistics in Table S1) generated by soaking the PrfA-DNA crystal in high 
A

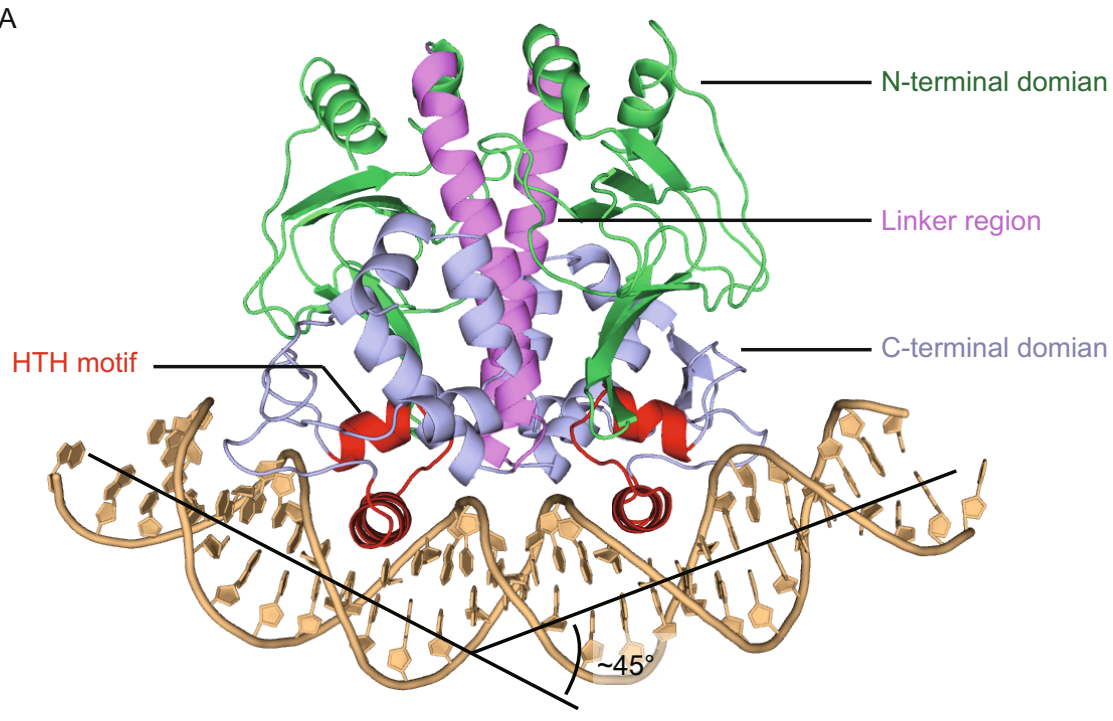

B

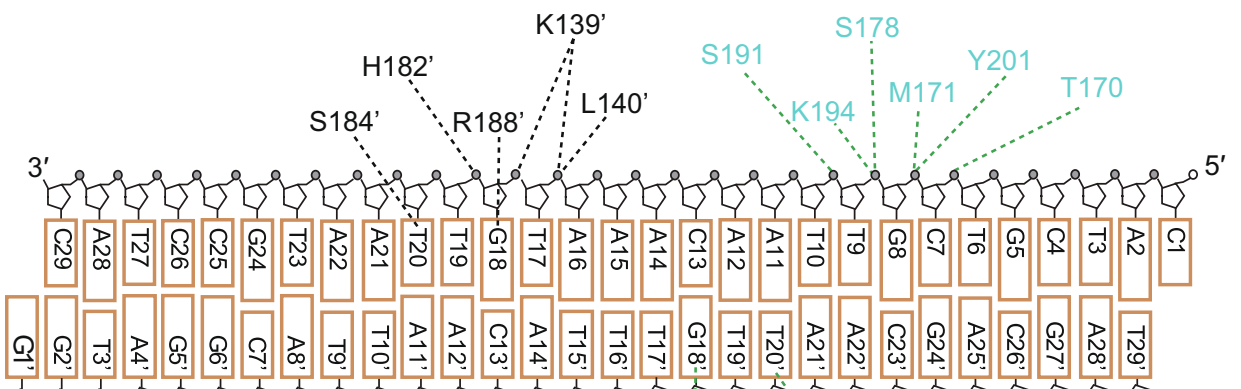

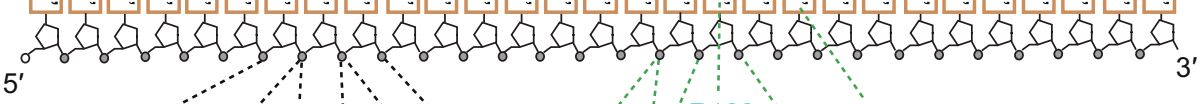

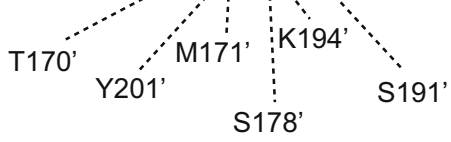

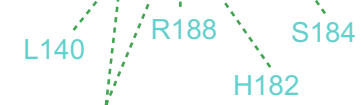

K139

C

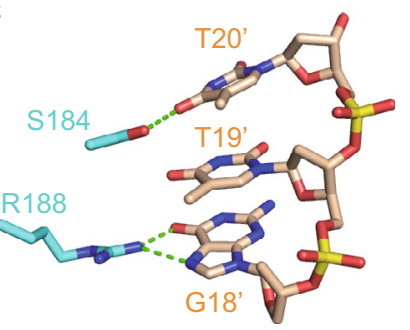

D

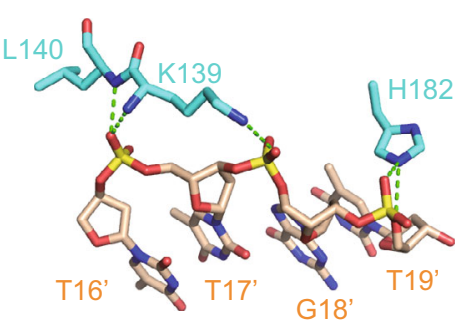

$\mathrm{F}$
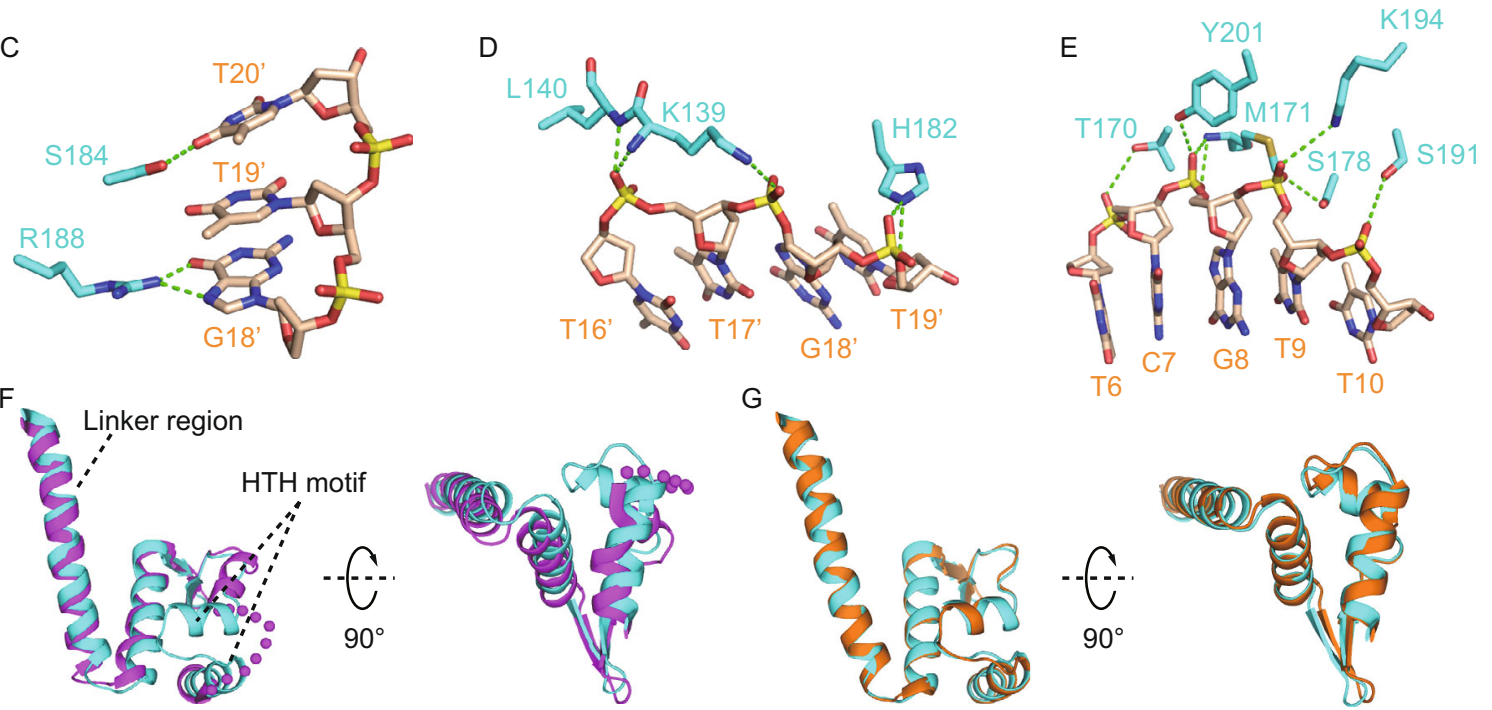

PrfA-DNA \& PrfA

PrfA-DNA \& PrfA ${ }^{6145 S}$ 
Figure 1. Structure of PrfA bound to DNA. (A) $2.93 \AA$ crystal structure of PrfA bound to a 28 bp DNA duplex (with one base $5^{\prime}$ overhang at each end). N-terminal domain, C-terminal domain, and linker region are colored in green, blue and violet, respectively. The helix-loop-helix $(\mathrm{HTH})$ motif in the C-terminal domain is colored in red. DNA is colored in light brown. The DNA is bent by appox. $45^{\circ}$ upon binding to PrfA. (B) Schematic of the detailed interactions between protein and DNA. The amino acids from two PrfA monomers are colored in cyan and black, respectively. (C-E) Hydrogen bonds interactions between one PrfA monomer with the DNA. ( $F$ and $G$ ) Superposed structures of PrfA in PrfA-DNA complex (cyan) with PrfA (WT) in free state (panel $F$, magenta) and PrfA ${ }^{G 145 S}$ in free state (panel G, brown). The disordered region in the structure of PrfA (WT) in free state is shown as magenta dots (panel F). The two panels are shown in the same view.

concentration glutathione solution. The overall structure of the ternary complex (Fig. 2A) is very similar to PrfA-DNA binary complex (Figs. $1 \mathrm{~A}$ and S1E), with no further conformational changes detected within either protein or DNA components following glutathione binding. The glutathione molecule binds into the central cleft surrounded by $\mathrm{N}$-terminal domain, C-terminal domain, and the $\alpha$-helical linker (Fig. 2A). Although the detailed interactions are different, the GSH binding sites in the PrfA-DNA complex are topographically equivalent to those for cAMP in Crp (Schultz et al., 1991) (Fig. S2A), CO in CooA (Lanzilotta et al., 2000) (Fig. S2B), OCPA in CprK (Levy et al., 2008) (Fig. S2C), and $2 O G$ in NtcA (Zhao et al., 2010) (Fig. S2D), indicating a conserved cofactor binding mode amongst the Crp/Fnr family members. The ternary complex structure also implies that the central ligand-binding cleft cannot accommodate the larger oxidized glutathione (GSSG), thereby providing a structural explanation for previous observation that PrfA does not bind to GSSG (Reniere et al., 2015). Complex formation between glutathione GSH and PrfA is mediated by van der Waals contacts and hydrogen bond interactions, whereby the glutathione molecule interacts with amino acids from both $\mathrm{N}$ - and $\mathrm{C}$-domains, as well as the linker region
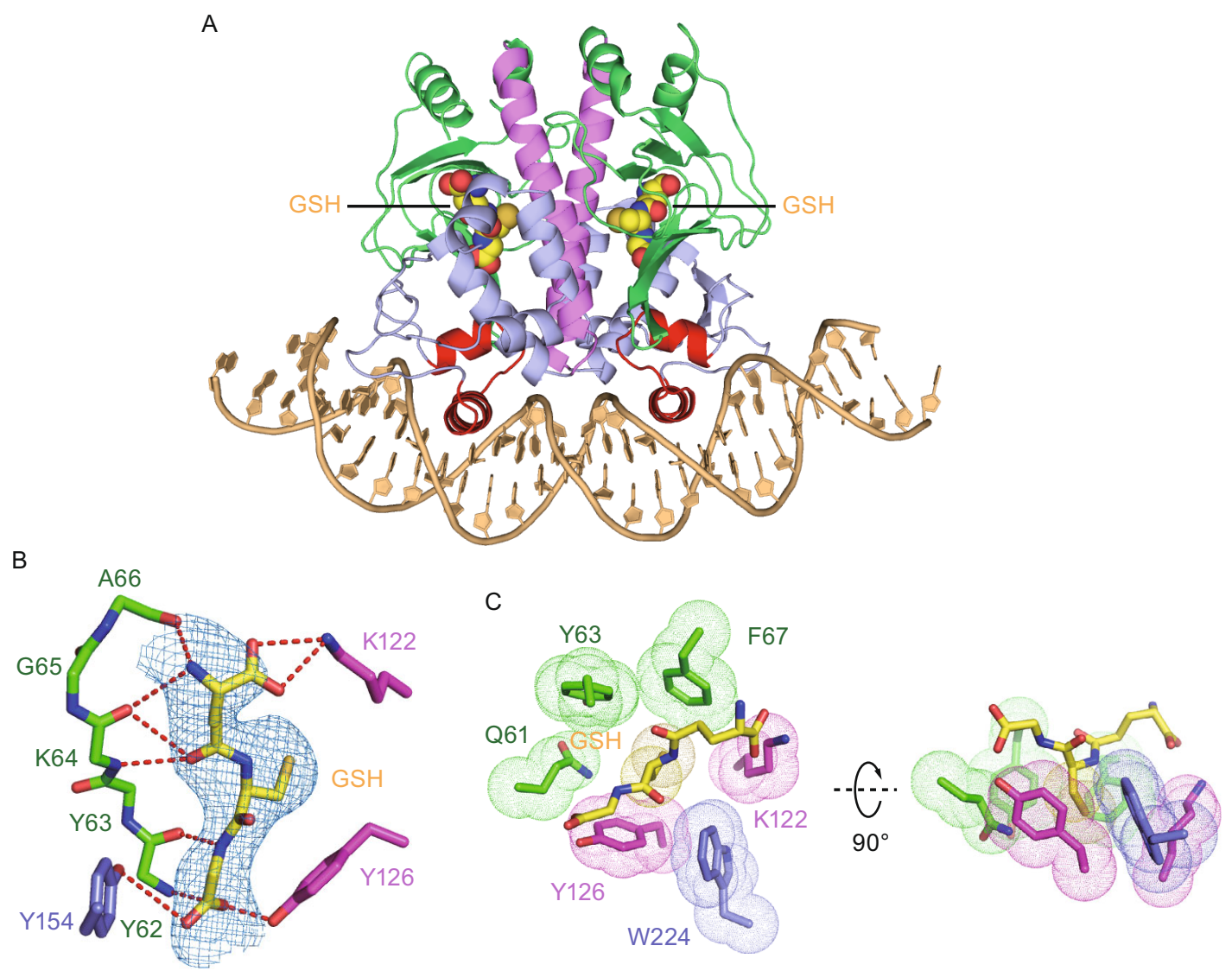

Figure 2. Structure of PrfA bound to DNA and glutathione. (A) $2.99 \AA$ crystal structure of PrfA bound to DNA and glutathione (GSH). The color code is same as in Fig. 1A. The glutathione is shown in a space filling representation. (B) Hydrogen bonds interactions between PrfA and GSH. The color code is same as in panel A. The 2Fo-Fc density of GSH in yellow is shown in blue mesh with $\sigma=1.0$. (C) The thiol group of GSH is embedded in a hydrophobic/aromatic pocket. The side chains of amino acids of PrfA and thiol group of GSH are shown in a dotted representation. 
(Fig. 2B and 2C). The hydrogen bonds are formed between glutathione with the main-chain of a $\beta$-strand (Y62-A66) in the $\mathrm{N}$-terminal domain, the side chain of $\mathrm{K} 122$ and $\mathrm{Y} 126$ from the linker region, and side chain of $\mathrm{Y} 154$ from C-terminal domain (Fig. 2C). The thiol group of glutathione is embedded in a hydrophobic/aromatic pocket composed of Q61, Y63, F67, K122, Y126, and W224 (Fig. 2C).

In this study, we have provided detailed structural information on PrfA bound to its target DNA sequence and cofactor glutathione GSH. We observed a DNA-induced conformational change of PrfA by comparing the structures of PrfA in free and DNA bound states (Figs. S1C and 1F). The conformation of PrfA in the PrfA-DNA and PrfA-DNAGSH complexes is similar to the constitutively active mutant PrfA ${ }^{\mathrm{G} 145 \mathrm{~S}}$ in free state (Fig. S1D and 1G), consistent with the previous prediction that $\operatorname{PrfA}^{\mathrm{G} 145 \mathrm{~S}}$ mutant adopts an induced conformation (Eiting et al., 2005). The DNA is bent by about $45^{\circ}$ upon complex formation with PrfA, which is smaller than the previously reported approx. $80^{\circ}$ to $90^{\circ}$ value for $\mathrm{Crp} / \mathrm{Fnr}$ family proteins: Crp and CprK (Benoff et al., 2002; Levy et al., 2008; Schultz et al., 1991). One possibility is that for PrfA-mediated gene regulation, the DNA does not need to be bent to the same degree observed for Crp and CprK complexes. Another possibility, however, is that the larger DNA bend in Crp and CprK complexes is due to the use of DNA molecules containing breaks, which may introduce artifacts during the crystallization process. In keeping with this hypothesis, an unpublished crystal structure of Crp-DNACAMP complex (RCSB: $3 \mathrm{MZH}$ ) shows the same degree of bending observed for our PrfA complexes when using an intact DNA lacking breaks (Fig. S1A). Interestingly, the DNA is bent slightly larger in our structures than in the recently reported PrfA-DNA complexes (Hall et al., 2016).

It has been proposed that PrfA activation constitutes a two-step process involving initial DNA binding followed by allosteric binding of glutathione for fully transcriptional activation (Reniere et al., 2015). This implies that glutathione binding will cause additional conformational change to the PrfA-DNA binary complex. However, we did not observe conformational differences between the PrfA-DNA binary complex and the PrfA-DNA-GSH ternary complex, which is consistent with the previous understanding that PrfA could interact with DNA in vitro even in the absence of an activator. In addition, recent structure determination of the $\operatorname{PrfA}^{\mathrm{G} 145 \mathrm{~S}}$. DNA complex (Hall et al., 2016) showed that there is no conformational difference between PrfA ${ }^{\text {G145S }}$-DNA and PrfADNA/PrfA-DNA-GSH complexes. Given that the constitutively active mutant $\operatorname{PrfA}{ }^{G 145 S}$ can completely bypass the requirement for glutathione during infection (Reniere et al., 2015), we conclude that our PrfA-DNA/PrfA-DNA-GSH structures represent the fully active conformation, the same as $\operatorname{PrfA}^{\mathrm{G} 145 \mathrm{~S}}$-DNA. More importantly, the recently solved structure of PrfA bound to GSH adopts the similar active conformation to PrfA-DNA-GSH complex, in contrast to the inactive conformation of the PrfA in free state (Hall et al., 2016). This indicates that the GSH-activated PrfA is primed for DNA binding (Hall et al., 2016). In line with the structural results, in vitro binding assays also confirmed that PrfA ${ }^{\mathrm{G} 145 \mathrm{~S}}$ (structurally equivalent to PrfA-GSH) shows stronger binding to the target DNA than the wild-type PrfA (Eiting et al., 2005). Based on the structural and biochemical results, we propose that glutathione will first bind to PrfA and induce local conformational change, which is a common feature of other Crp/Fnr family members. Next, GSH-bound PrfA will bind to the target DNA to regulate gene transcription.

\section{FOOTNOTES}

This work was supported by the National Natural Science Foundation of China (Grant No. 31670903), the start-up funding of Institute of Biophysics, and the CAS Center for Excellence in Biomacromolecules to P.G. The atomic coordinates and structure factors have been deposited in the Protein Data Bank with PDB ID codes 5X6D (PrfA-DNA) and 5X6E (PrfA-DNA-GSH). Yong Wang, Han Feng, Yalan Zhu, and $\mathrm{Pu}$ Gao declare that they have no conflict of interest. This article does not contain any studies with human or animal subjects performed by the any of the authors.

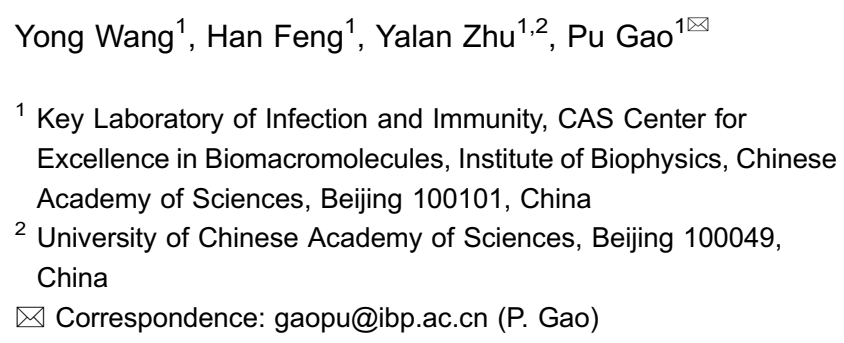

\section{OPEN ACCESS}

This article is distributed under the terms of the Creative Commons Attribution 4.0 International License (http://creativecommons.org/ licenses/by/4.0/), which permits unrestricted use, distribution, and reproduction in any medium, provided you give appropriate credit to the original author(s) and the source, provide a link to the Creative Commons license, and indicate if changes were made.

\section{REFERENCES}

Benoff B, Yang H, Lawson CL, Parkinson G, Liu J, Blatter E, Ebright YW, Berman HM, Ebright RH (2002) Structural basis of transcription activation: the CAP-alpha CTD-DNA complex. Science 297:1562-1566

Chakraborty T, Leimeister-Wachter M, Domann E, Hartl M, Goebel W, Nichterlein T, Notermans S (1992) Coordinate regulation of virulence genes in Listeria monocytogenes requires the product of the prfA gene. J Bacteriol 174:568-574

Eiting M, Hageluken G, Schubert WD, Heinz DW (2005) The mutation G145S in PrfA, a key virulence regulator of Listeria monocytogenes, increases DNA-binding affinity by stabilizing the HTH motif. Mol Microbiol 56:433-446

Freitag NE, Port GC, Miner MD (2009) Listeria monocytogenesfrom saprophyte to intracellular pathogen. Nat Rev Microbiol 7:623-628 
Freitag NE, Rong L, Portnoy DA (1993) Regulation of the prfA transcriptional activator of Listeria monocytogenes: multiple promoter elements contribute to intracellular growth and cell-tocell spread. Infect Immun 61:2537-2544

Hall M, Grundstrom C, Begum A, Lindberg MJ, Sauer UH, Almqvist F, Johansson J, Sauer-Eriksson AE (2016) Structural basis for glutathione-mediated activation of the virulence regulatory protein PrfA in Listeria. Proc Natl Acad Sci USA 113:14733-14738

Hansen K, Prabakaran T, Laustsen A, Jorgensen SE, Rahbaek SH, Jensen SB, Nielsen R, Leber JH, Decker T, Horan KA et al (2014) Listeria monocytogenes induces IFNbeta expression through an IFI16-, cGAS- and STING-dependent pathway. EMBO J 33:1654-1666

Krawczyk-Balska A, Markiewicz Z (2015) The intrinsic cephalosporin resistome of Listeria monocytogenes in the context of stress response, gene regulation, pathogenesis and therapeutics. J Appl Microbiol 120:251-265

Lanzilotta WN, Schuller DJ, Thorsteinsson MV, Kerby RL, Roberts GP, Poulos TL (2000) Structure of the CO sensing transcription activator CooA. Nat Struct Biol 7:876-880

Leimeister-Wachter M, Haffner C, Domann E, Goebel W, Chakraborty $T$ (1990) Identification of a gene that positively regulates expression of listeriolysin, the major virulence factor of Listeria monocytogenes. Proc Natl Acad Sci USA 87:8336-8340

Levy C, Pike K, Heyes DJ, Joyce MG, Gabor K, Smidt H, van der Oost J, Leys D (2008) Molecular basis of halorespiration control by CprK, a CRP-FNR type transcriptional regulator. Mol Microbiol 70:151-167

Rayamajhi M, Humann J, Penheiter K, Andreasen K, Lenz LL (2010) Induction of IFN-alphabeta enables Listeria monocytogenes to suppress macrophage activation by IFN-gamma. J Exp Med 207:327-337

Reniere ML, Whiteley AT, Hamilton KL, John SM, Lauer P, Brennan RG, Portnoy DA (2015) Glutathione activates virulence gene expression of an intracellular pathogen. Nature 517:170-173

Schultz SC, Shields GC, Steitz TA (1991) Crystal structure of a CAPDNA complex: the DNA is bent by 90 degrees. Science 253:1001-1007

Zhao MX, Jiang YL, He YX, Chen YF, Teng YB, Chen Y, Zhang CC, Zhou CZ (2010) Structural basis for the allosteric control of the global transcription factor NtcA by the nitrogen starvation signal 2-oxoglutarate. Proc Natl Acad Sci USA 107:12487-12492

Yong Wang and Han Feng have contributed equally to this work.

Electronic supplementary material The online version of this article (doi:10.1007/s13238-017-0390-x) contains supplementary material, which is available to authorized users. 